\section{Long life still hard on the brain}

$\mathrm{T}$ he average life expectancy in the US has hit a record high of 77.6 years. The Centers for Disease Control attribute this statistic to declining death rates from heart disease, cancer, stroke, HIV/AIDS, drug abuse, and firearms. But as the aging population increases, so does the risk for age-related syndromes such as Parkinson disease (PD) and Alzheimer disease (AD), for which there are no cures. Recent research offers a better understanding of the etiology of these neurodegenerative diseases and hope for future treatment. In the February 25, 2005, issue of Science, researchers discovered axonal defects that precede known AD pathology - swellings along axons containing cellular motor proteins, organelles, and vesicles (1). Hindering the axonal motor transport machinery amplified the frequency of these defects and increased amyloid deposits in the brains of mice with AD. These findings indicate that decreased axonal transport might promote the formation of senile plaques characteristic of AD. Another study, published in the April issue of the American Journal of Human Genetics, shows that a novel mutation in the leucine-rich repeat kinase 2 (LRRK2) gene causes a genetic component of PD in several families (2). These new insights into the pathophysiology of age-related neurodegenerative diseases will help ease the burden on the growing elderly population.

1. Stokin, G.B., et al. 2005. Axonopathy and transport deficits early in the pathogenesis of Alzheimer's disease. Science. 307:1282-1288.

2. Kachergus, J., et al. 2005. Identification of a novel LRRK2 mutation linked to autosomal dominant parkinsonism: evidence of a common founder across European populations. Am. J. Hum. Genet 76:672-680.

\section{Stacie Bloom}

\title{
Taking a (mosquito) bite out of malaria
}

arly in the last decade, great strides were made toward eliminating malaria through the use of pest control and extermination of mosquitoes, which are responsible for transmitting the infectious Plasmodium parasites that cause the disease. Malaria was once prevalent in the US and Europe, but the draining of wetlands and improved sanitation eliminated the scourge from these affluent regions. Today, most deaths from the disease occur in poor, rural areas of Africa, where malaria incidence is on the rise, killing more than one million people each year, most of whom are young children. This statistic seems shocking considering the disease's preventability by relatively simple means, such as mosquito netting and prophylactic drugs such as quinine and its derivatives.

Unfortunately, pharmaceutical treatment is costly, in short supply, and rendered ineffective as more virile strains of the disease become resistant to the medication, so malaria prevention is widely considered a top priority. As a first line of defense, the United Nations Children's Fund recommends the wide distribution of mosquito nets impregnated with insect repellents as the most cost-effective disease prevention method. Many international health organizations have now donated millions of dollars for the production and allocation of insecticide-treated nets. The United Kingdom's Department for International Development's \$3 million donation will provide 400,000 of these nets to pregnant women and children less than 5 years of age in Zimbabwe, where malaria is the second-highest killer of young children. Japan, in an unprecedented contribution, has promised 10 million bed nets to Africa by 2007. The Global Fund, through US sponsorship, began a \$22 million, 5-year plan to fight malaria in 2003 at the urging of Swaziland, Mozambique, and South Africa. According to the Global Fund, the efforts have "tripled the area of operations in Mozambique.” The US pledges at least $\$ 2$ billion more through 2008.

The WHO's Roll Back Malaria Department has attempted to strike preemptively by initiating the Malaria Early Warning System, which began monitoring rainfall in June 2002. Rainfall in warm, semi-arid and desert-

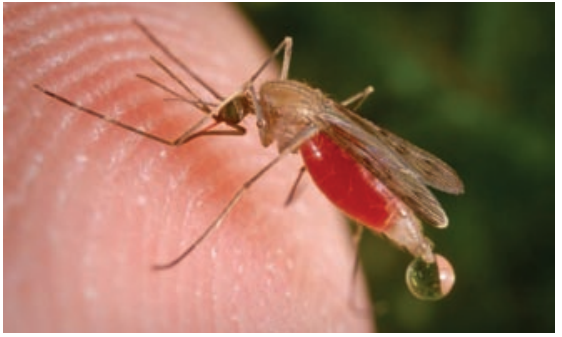

This mosquito is taking a blood meal from a human host. During blood meals taken from persons infected with malaria, mosquitoes pick up malaria parasites. The parasites can be introduced into another human host when the mosquito takes another blood meal. Image courtesy of James Gathany (Centers for Disease Control, Atlanta, Georgia, USA).

fringe regions can trigger explosive epidemics by providing fertile breeding grounds for mosquitoes. By studying rainfall data, the organization is able to identify regions at risk for a future epidemic, and assistance can arrive before the outbreak occurs.

On the clinical front, technological advances have paved the way for new means of malaria prevention. Using the recently decoded genomes of both the carrier mosquito and the dangerous parasite it spreads, scientists hope to exploit sequences that might belie weaknesses in the genetic makeup of the organisms in order to disrupt the life cycle of the deadly protozoan. Researchers have already found genes that can be manipulated to help produce more effective repellents and insecticides. Theoretically, the pests themselves can be genetically altered to resist malaria, but that idea invites controversy, with the possibility of genetic mixing among mosquito species perhaps creating an even stronger malaria carrier.

Controversy aside, the world is witnessing an increasing effort to eradicate the disease by boosting prevention measures and identifying new treatment options. The alarming number of child-related deaths from this wholly treatable illness has signaled the urgency to treat malaria on a global scale. The donations by world bodies, innovative research by both public and private organizations, and new advancement in technology represent fresh hope.

\section{Rick Ring}

